\title{
The role of high sensitive C-reactive protein and histopathological evaluation in chronic gastritis patients with or without Helicobacter pylori infection ${ }^{1}$
}

\author{
Eren Altun' ${ }^{\oplus}$, Ayla Yildiz", Celalettin Cevik'", Gulay Turan'
}

\begin{abstract}
'Assistant Professor, Balikesir Universty, Faculty of Medicine, Department of Pathology, Balikesir, Turkey. Scientific, intellectual, conception and design of the study; acquisition of data; technical procedures; histopathological examinations; manuscript preparation and writing; critical revision.

"Assistant Professor, Balikesir Universty, Faculty of Medicine, Department of Gastroenterology, Balikesir, Turkey. Conception and design of the study.

I'Assistant Professor, Balikesir University, Faculty of Health Sciences, Balikesir, Turkey. Acquisition, analysis and interpretation of data; histopathological examinations; statistics analysis.
\end{abstract}

\begin{abstract}
Purpose: To evaluate serum levels of high-sensitivity C-reactive protein (hs-CRP) in chronic gastritis patients to predict Helicobacter pylori (HP) infection, inflammatory activity, and precancerous lesions.

Methods: A total of 811 patients with upper gastrointestinal symptoms and histopathological diagnosis of chronic gastritis were enrolled in the study. On endoscopy, five gastric biopsies were taken according to Modified Sydney protocol, which were stained with hematoxylin \& eosin and Giemsa

Results: HP infection was found in $28.6 \%$ of patients, being significantly more common in specimens with acute and chronic inflammatory activity. Mucosal atrophy, intestinal metaplasia, and dysplasia were found in $20.2 \%, 18.8 \%$ and $2.7 \%$ of biopsy specimens. Mean hs-CRP was $1.9 \pm 1.6 \mathrm{mg} / \mathrm{dl}$ for males and $2.2 \pm 1.9 \mathrm{mg} / \mathrm{dl}$ for females. hs-CRP average were significantly higher in patients with severe acute inflammation (p:0.049), in patients with severe chronic inflammation ( $p: 0.015)$ and in those with HP ( $p: 0.001)$. The severity of HP infection increased significantly with the increased degree of acute inflammation, chronic inflammation and hs-CRP level ( $p=0.001$ for both).

Conclusion: Serum hs-CRP level increases in patients with chronic gastritis, it could be an indicator of severity of acute or chronic mucosal inflammation, and presence of HP infection. Therefore, hs-CRP may aid the diagnosis of chronic gastritis, but it is not associated with precancerous lesions.
\end{abstract}

Key words: Gastritis. C-Reactive Protein. Helicobacter pylori. Inflammation. 


\section{- Introduction}

Helicobacter pylori (HP) affects over half of the population worldwide and is the major etiology of chronic gastritis ${ }^{1}$. It colonizes in gastric mucosa and causes chronic mucosal inflammation leading to gastritis. $\mathrm{HP}$-associated chronic gastritis is considered precancerous and has a high potential of progression into gastric cancer unless effectively treated ${ }^{2}$. The histolopathological evidence of precancerous lesions of chronic gastritis, such as gastric atrophy and intestinal metaplasia has been well known to be associated with HP infection ${ }^{3,4}$.

Since it is an infection causing an inflammatory disease, HP gastritis is associated with the increase in serum markers of inflammation such as interleukin family, C-reactive protein (CRP), platelets, neutrophils ${ }^{5-8}$. Among these markers, CRP is an acute-phase protein, which is commonly used as a marker of various systemic acute and chronic inflammatory conditions ${ }^{9}$. As serum level of CRP is normally less than $1 \mathrm{mg} / \mathrm{dl}$, it can increase up to $35-40 \mathrm{mg} / \mathrm{dl}$ in response to infection or inflammation. Upon understanding its value in diagnosis of various conditions, high-sensitivity CRP (hs-CRP) assay has been developed to measure CRP in a sensitivity of $0.5 \mathrm{mg} / \mathrm{dl}^{10}$. In addition to being an indicator of inflammation, hs-CRP has been suggested to be a marker of cardiovascular diseases and gastric cancer in patients with HP-associated chronic gastritis ${ }^{6,11}$. Although serum level of hs-CRP has been known to increase in chronic gastritis with HP infection, limited studies have focused on the association of hs-CRP level with inflammatory activity and presence of precancerous lesions in biopsy specimens ${ }^{11,12}$. If hs-CRP level is predictive of inflammation level and precancerous lesions, it can be used as a simple and easily accesible laboratory test in early diagnosis, treatment response monitoring, and cancer screening in chronic gastritis patients.

Therefore, in this study we aimed to determine serum levels of hs-CRP in chronic gastric patients with prediction of HP infection and precancerous lesions in a large series of 811 patients. We also investigated the association of severity of HP infection with hs-CRP level and chronic inflammation activity.

\section{Methods}

\section{Ethics approval and consent to participate}

In this study, the investigation protocol was in accordance with the Helsinki Committee requirement and was approved by the institutional ethical committee of Balikesir University (Decision no: 2018/101, 2018/05/09). Additional patient consent for this clinical research was not required.

\section{Study population}

A total of 811 patients who applied to our clinic with upper gastrointestinal symptoms suggestive of chronic gastritis and underwent endoscopic examination between 2014 and 2017 were enrolled in this study. Patients having active infection, tumor, other gastrointestinal diseases, severe systemic diseases, using any drug with potential effect on gastrointestinal or coagulation system, and pregnant or breastfeeding women were excluded from study.

\section{Endoscopy, histopathology and biochemistry}

The data regarding for this retrospective research study has been obtained from Balikesir University Hospital. For endoscopic examination, standard gastrointestinal video 
endoscopy was performed under sedation and local anesthesia of pharyngeal mucosa. Esophagus, stomach and duodenum were evaluated for the visible abnormal findings and lesions, and four gastric biopsies specimens were taken from antrum and corpus for histopathological diagnosis of gastritis and detection of HP. Biopsy specimens were stained with hematoxylin eosin (H\&E) and Giemsa, and evaluated for histopathology and HP under light microscope (Fig. 1).

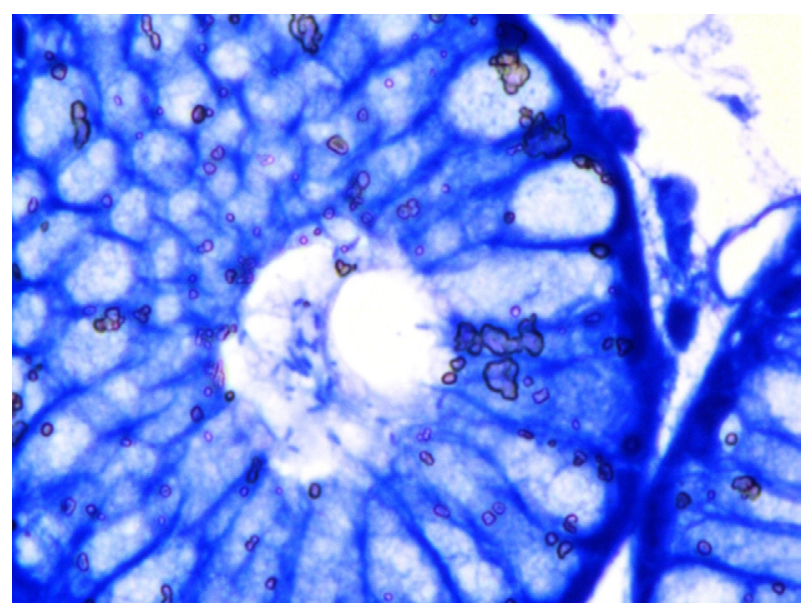

Figure 1 - Helicobacter pylori (HP) bacilli seen at the center of gland (Giemsa, x100).

Histopathological findings were defined and classified according to updated Sydney system $^{3}$. Accordingly, gastritis is defined as the atropic or non-atropic inflamation of the gastric mucosa. The presence of mucosal atrophy, which is the loss of specialized glands and decrease in the thickness of mucosa; intestinal metaplasia, which is the metaplastic transformation of gastric glandular and surface epithelium towards intestinal mucosal elements; dysplasia, which is the neoplastic transformation of epithelial cells were evaluated. Acute inflammation or active disease was defined as neutrophilic infiltrates of the lamina propria, pits, or surface epithelium. It was graded as mild if less than one third of pits and surface was infiltrated, moderate if one third to two thirds was infiltrated, and severe if more than two third was infiltrated according the Modified Sydney Protocol. Chronic inflammation was defined as increased lymphocytes and plasma cells in the lamina propria, and graded as mild, moderate or severe according to the density of increase. HP density was defined as mild if organism covered less than one third of the surface, severe if there were larger clusters or a continuous layer over two thirds of surface, moderate if density was somewhere between mild and severe HP density. hs-CRP were measured by standard laboratory methods from intravenous blood sample of each patient.

\section{Biochemistry}

White blood cell count (WBC), platelet count (PC), lymphocyte count (LC), and hsCRP were measured by standard laboratory methods from intravenous blood sample of each patient. $L C / W B C$ ratio and $P C / W B C$ ratio were calculated as an indicator of HP infection and outcome of patients, respectively.

\section{Statistical analysis}

The study data were summarized by descriptive statistics (e.g., mean, standart deviation, frequency, percentage). Student's $t$ test or analysis of variance (ANOVA) was used to evaluate the significance of difference in serum hs-CRP level between two or more than two subgroups of study parameters. For comparison of qualitative data between histological severity groups of HP infection, chi-square test was used. Logistic regression analysis was used to evaluate the association of HP with chronic inflammation activity and 
serum hs-CRP level. A p value smaller than 0.05 was considered statistically significant.

\section{Results}

On histopathological evaluation, acute and chronic inflammation in any severity was detected in $31.4 \%$ and $64 \%$ of patients, respectively. Of 811 patients, $28.6 \%$ had HP infection. Mucosal atrophy, intestinal metaplasia, and dysplasia were found in $20.2 \%$, $17.8 \%$, and $2,7 \%$ of biopsy specimens obtained from 811 patients.

\section{Serum hs-CRP levels}

Mean hs-CRP was $1.9 \pm 1.6 \mathrm{mg} / \mathrm{dl}$ for males and $2.2 \pm 1.9 \mathrm{mg} / \mathrm{dl}$ for females. hs-CRP average; ( $p$ : 0.009) were significantly higher in patients with severe acute inflammation (p: 0.049), in patients with severe chronic inflammation ( $p: 0.015)$ and in those with HP ( $p$ : 0.001). Presence of mucosal atrophy, intestinal metaplasia or dysplasia had no significant effect on serum hs-CRP level $(p>0.05)$ (Table 1).

Table 1 - Demographic and clinical characteristics of study patients $(n=811)$.

\begin{tabular}{|c|c|c|c|c|}
\hline & & & $\mathrm{s}-\mathrm{CRP} / \mathrm{mg} / \mathrm{d}$ & \\
\hline & & n (\%) & Mean \pm SD & p value \\
\hline Gender & Male & $361(44.5)$ & $1.9 \pm 1.6$ & คค०१ а \\
\hline & Female & $450(55.5)$ & $2.2 \pm 1.9$ & $0.009^{\circ}$ \\
\hline Years & $18-45$ & $538(66.3)$ & $2.3 \pm 1.8$ & $0.006^{a}$ \\
\hline & $46+$ & $273(33.7)$ & $1.8 \pm 1.6$ & \\
\hline Acute inflammation & None & $556(68.6)$ & $2.1 \pm 1.7$ & \\
\hline & Mild & $153(18.9)$ & $2.0 \pm 1.8$ & 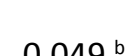 \\
\hline & Moderate & $53(6.5)$ & $2.3 \pm 1.8$ & 0.045 \\
\hline & Severe* & $49(6.0)$ & $2.8 \pm 1.9$ & \\
\hline Chronic inflammation & None & $292(36.0)$ & $2.2 \pm 1.8$ & \\
\hline & Mild & $288(35.5)$ & $1.9 \pm 1.7$ & 0.15 b \\
\hline & Moderate & $149(18.4)$ & $2.0 \pm 1.6$ & U.U15 \\
\hline & Severe* & $82(10.1)$ & $2.6 \pm 2.0$ & \\
\hline HP infection & Yes & $232(28.6)$ & $2.5 \pm 1.9$ & คดค1 а \\
\hline & No & $579(71.4)$ & $2.0 \pm 1.7$ & 0.001 \\
\hline Mucosal atrophy & Yes & $164(20.2)$ & $2.1 \pm 1.7$ & 0511 a \\
\hline & No & $647(79.8)$ & $2.1 \pm 1.8$ & 0.511 \\
\hline Intestinal metaplasia & Yes & $144(17.8)$ & $2.0 \pm 1.7$ & 0117 а \\
\hline & No & $667(82.2)$ & $2.1 \pm 1.8$ & 0.417 \\
\hline Dysplasia & Yes & $22(2.7)$ & $2.0 \pm 1.7$ & 0591 a \\
\hline & No & $789(97.3)$ & $2.1 \pm 1.8$ & 0.591 \\
\hline
\end{tabular}

a Student's t test, ${ }^{\mathrm{b}}$ ANOVA

hs-CRP, high sensitive C-reactive protein; SD, standard deviation. 


\section{HP infection}

When histological severity of HP subgroups were compared for study parameters, we found that severity of HP infection increased significantly with the increased degree of acute inflammation, chronic inflammation and hs-CRP level
( $p=0.001$ for both). We found that severity of HP infection decreased significantly with the increased patient's age. (p:0.030) (Table 2).

On the other hand, severity subgroups of HP or the presence of HP infection did not show significance difference with gender, mucosal atrophy, intestinal metaplasia, and dysplasia ( $p>0.05$ ) (Table 2).

Table 2 - Severity of HP infection with respect to study parameters $(n=811)$.

\begin{tabular}{|c|c|c|c|c|c|c|}
\hline & \multicolumn{6}{|c|}{ Histological severity of HP infection } \\
\hline & & $\begin{array}{c}\text { None } \\
(n=708)\end{array}$ & $\begin{array}{c}\text { Mild } \\
(n=109)\end{array}$ & $\begin{array}{c}\text { Moderate } \\
\qquad(n=99)\end{array}$ & $\begin{array}{l}\text { Severe } \\
(n=58)\end{array}$ & $\begin{array}{c}p \\
\text { value }\end{array}$ \\
\hline \multirow[t]{2}{*}{ Gender } & Male & $251(69.5)$ & $51(14.1)$ & $38(10.5)$ & $21(5.8)$ & \multirow{2}{*}{$0.159^{a}$} \\
\hline & Female & $328(72.9)$ & $41(9.1)$ & $50(11.1)$ & $31(6.9)$ & \\
\hline \multirow[t]{2}{*}{ Years } & $18-45$ & $183(67.0)$ & $31(11.4)$ & $42(15.4)$ & $17(6.2)$ & \multirow[t]{2}{*}{$0.030^{\mathrm{a}}$} \\
\hline & $46+$ & $396(73.6)$ & $61(11.3)$ & $46(8.6)$ & $35(6.5)$ & \\
\hline \multirow[t]{4}{*}{ Acute inflammation } & None & $474(85.3)$ & $38(6.8)$ & $24(4.3)$ & $20(3.6)$ & \multirow{4}{*}{$0.001^{a}$} \\
\hline & Mild & $75(49.0)$ & $29(19.0)$ & $34(22.2)$ & $15(9.8)$ & \\
\hline & Moderate & $18(34.0)$ & $13(24.5)$ & $15(28.3)$ & $7(13.2)$ & \\
\hline & Severe & $12(24.5)$ & $12(24.5)$ & $15(30.6)$ & $10(20.4)$ & \\
\hline \multirow[t]{4}{*}{ Chronic inflammation } & None & $283(96.9)$ & $6(2.1)$ & $3(1.0)$ & $0(0.0)$ & \multirow{4}{*}{$0.001^{a}$} \\
\hline & Mild & $190(66.0)$ & $35(12.2)$ & $37(12.8)$ & $26(9.0)$ & \\
\hline & Moderate & $81(54.4)$ & $31(20.8)$ & $20(13.4)$ & 17 (11.4) & \\
\hline & Severe & $25(30.5)$ & $20(24.4)$ & $28(34.1)$ & 9 (11.0) & \\
\hline \multirow[t]{2}{*}{ Mucosal atrophy } & Yes & $113(68.9)$ & $20(12.2)$ & $15(9.1)$ & $16(9.8)$ & \multirow{2}{*}{$0.217^{\mathrm{a}}$} \\
\hline & No & $466(72.0)$ & $72(11.1)$ & $73(11.3)$ & $36(5.6)$ & \\
\hline \multirow[t]{2}{*}{ Intestinal metaplasia } & Yes & $97(67.4)$ & $16(11.1)$ & $23(16.0)$ & $8(5.6)$ & \multirow{2}{*}{$0.184^{\mathrm{a}}$} \\
\hline & No & $482(72.3)$ & $76(11.4)$ & $65(9.7)$ & $44(6.6)$ & \\
\hline \multirow[t]{2}{*}{ Dysplasia } & Yes & $17(77.3)$ & $3(13.6)$ & $2(9.1)$ & $0(0.0)$ & \multirow{2}{*}{$0.185^{a}$} \\
\hline & No & $562(71.2)$ & $89(11.3)$ & 86 (10.9) & $52(6.6)$ & \\
\hline hs-CRP (mg/dl) & mean $\pm S D$ & $1.2 \pm 0.7$ & $2.1 \pm 0.6$ & $2.5 \pm 0.2$ & $2.8 \pm 0.3$ & $0.001^{b}$ \\
\hline
\end{tabular}

${ }^{\mathrm{a}}$ Chi-square test, ${ }^{\mathrm{b}}$ ANOVA

SD: Standart Deviation

Association of HP infection with chronic inflammation and hs-CRP level

According to the multiple regression analysis using the Backward model, gender, age, and severity of HP significantly affect hs-CRP levels, whereas acute inflammation, chronic inflammation, and intestinal metaplasia did not affect hs-CRP levels (Table 3). 
Table 3 - Evaluation of the relationship between hs-CRP ( $\mathrm{mg} / \mathrm{dl}$ ) levels and demographic and clinical findings.

\begin{tabular}{lccccccc} 
& & \multicolumn{3}{c}{$\begin{array}{c}\text { Standardized } \\
\text { Coefficients }\end{array}$} & $\mathbf{t}$ & $\mathbf{p}$ & \multicolumn{3}{c}{$\begin{array}{c}95.0 \% \text { Confidence } \\
\text { Interval for B }\end{array}$} \\
& Beta & Std. Error & Beta & & & Lower & Upper \\
\hline (Constant) & 2.305 & 0.116 & & 19.835 & 0.000 & 2.077 & 2.534 \\
Gender & -0.317 & 0.126 & -0.087 & -2.525 & $\mathbf{0 . 0 1 2}$ & -0.564 & -0.071 \\
Years & -0.405 & 0.133 & -0.106 & -3.046 & $\mathbf{0 . 0 0 2}$ & -0.666 & -0.144 \\
Acute inflammation & 0.106 & 0.102 & 0.051 & 1.042 & 0.298 & -0.094 & 0.307 \\
Chronic inflammation & -0.064 & 0.091 & -0.034 & -0.698 & 0.485 & -0.243 & 0.115 \\
Severity of HP & 0.280 & 0.076 & 0.143 & 3.669 & $\mathbf{0 . 0 0 1}$ & 0.130 & 0.429 \\
Intestinal metaplasia & -0.186 & 0.167 & -0.039 & -1.115 & 0.265 & -0.514 & 0.142 \\
\hline
\end{tabular}

F:5.781 (p:0.001).

HP status and effective factors were evaluated by logistic regression model. It is observed that the model ( $c 2=676.957$, $p=0.001$, Nagelkerke R2 $=0.41$ ) is to significant. Sex, acute inflammation, chronic inflammation, and hs-CRP were statistically significant in the model. The correct classification rate of the model in general is $84.2 \%$. According to these results, it is seen that the established model is a valid and usable model.

The Presence of HP in those aged between the age of 18 and 45 is 1.72 times (1.15-2.55) higher than those aged 46 years and upper.

The presence of HP is higher in patients with low acute inflammation was 2.63 times (1.68-4.11) in patients with moderate acute inflammation 4.2 times (2.0-8.8), and in patients with severe acute inflammation 6.1 times (2.5-14.9) when compared with those without acute inflammation.

The presence of HP is higher in patients with low levels of chronic inflammation 13.0 times (6.25-27.33), in those with moderate chronic inflammation 13.1 times (5.84-29.52), in patients with severe chronic inflammation 17 times (6.40-45.60) when compared with those without chronic inflammation. The possibility of the presence of HP increases
1.19 times, when 1 unit increases in hs-CRP level.

\section{Discussion}

In this extensive retrospective research study, we examined endoscopic biopsy specimens and serum hs-CRP levels in 811 patients. We found that in patients acute inflammation, chronic inflammation, and HP infection, hs-CRP levels were above normal levels. As acute and chronic inflammation increases, the level of hs-CRP increases. However, we have indicated that precancerous lesions, metaplasia and atrophy are not associated with the level of hs-CRP.

We determined in our study population with chronic gastritis that the mean of hs-CRP was $1.9 \pm 1.6 \mathrm{mg} / \mathrm{dl}$ for males and $2.2 \pm 1.9 \mathrm{mg} /$ $\mathrm{dl}$ for females, which was higher $1 \mathrm{mg} / \mathrm{dl}$ than the normal hs-CRP level ${ }^{9}$. Consistent with to the studies in literature, it is found increased hs-CRP levels in parallel to the severity of HP gastritis compared to gastritis without HP infection in our study, ${ }^{6,12}$. In adition, we also found that serum hs-CRP level of chronic gastritis patients with HP infection was significantly higher than those without HP infection and there was significant association 
between severity of HP infection and hs-CRP level. We also found that serum hs-CRP levels have been affected by the severity of acute or chronic inflammation. Furthermore, Raut et al. ${ }^{6}$ evaluated 80 patients and found that patients with chronic gastritis and HP infection was higher hs-CRP levels than chronic gastritis patients without HP. Similarly in a study of 239 patients with chronic gastritis, Rahmani et al. ${ }^{12}$ reported that serum hs-CRP levels of patients with HP gastritis was higher than those without HP and hs-CRP was positively correlated with mucosal inflammatory activity and dysplasia.

Although several staining methods have been suggested to determine the severity of HP infection ${ }^{13,14}, \mathrm{H} \& \mathrm{E}$, alone or in combination with other methods, is the standard staining technique to detect the presence and extent of infection ${ }^{3,15}$. Thus, in the present study, we used the standard H\&E staining and Giemsa staining for histopathological evaluation and HP assessment of biopsy materials. In our study population, chronic gastritis was mostly associated with HP infection. It has been known that the presence of acute or chronic inflammatory gastritis is highly predictive of active HP infection in correlation with the severity of inflammation ${ }^{14}$. Similarly, in our study, severity of HP infection increased with the increased severity of acute or chronic inflammation, and in ordinal logistic regression analysis, HP was associated with degree of chronic inflammation activity.

In clinical studies and meta-analysis, eradication of HP decreases the development of gastric cancer in chronic gastritis patients with precancerous lesions such as atrophy, metaplasia, and displasia ${ }^{16,17}$. In addition to causing gastric cancer, HP gastritis has been claimed to induce endothelial dysfunction and potentially to cause cardiovascular pathologies ${ }^{6}$. For early diagnosis against HP and precancerous lesions, histopathological diagnosis of gastritis along with gastric cancer screening is of great importance. In order to standardize the histopathologic diagnosis and classification of gastritis, the Sydney System updated in 1994 which has been commonly used in practice and clinical studies ${ }^{3}$. The updated Sydney System highlighted the importance of combining etiology, topography and histology of gastritis for the best clinical management of the disease $e^{3,18,19}$. However, there has been limited studies on the gastric cancer screening in chronic gastritis patients with HP infection. Chung et al. ${ }^{11}$ reported that hs-CRP levels can be used in combination with other serum markers for screening of gastric cancer in patients with chronic gastritis. Rahmani et al. ${ }^{12}$ found that although the presence of atrophy or metaplasia did not affect serum hs-CRP levels, gastritis patients having dysplasia had significantly higher hs-CRP level than patients without dysplasia. In our study, mucosal atrophy, intestinal metaplasia and dysplasia have no effect on serum hs-CRP level. In contrary to previous studies ${ }^{3,4}$, we found that there is no relation between the presence of any precancerous lesion and severity of HP infection.

Moreover, $\mathrm{H}$. Pylori is one of the most prevalant chronic infection diseases in the World and previous studies have been clearly demonstrated that there was closely association between peptic ulcer, chronic gastritis, gastric cancer and mucosa associated tissue lym phoma $(M A L T)^{20}$. On the other hand, it is known that the presence of extragastrointestinal diseases including coronary arter diseases and diabetes mellitus were higher in patient with $\mathrm{H}$. Pylori infection $^{21}$. In light of these knowledge, the presence of severe $\mathrm{H}$. Pylori infection may accompany high hsCRP levels.

\section{- Conclusions}

Inconclusion, intheliterature, commonly serum hs-CRP level have been claimed that it 
can be used as a simple and easily accesible laboratory test to predict inflammatory activity and precancerous lesions. Thus for treatment response monitoring and cancer screening in chronic gastritis patients could be easy. In this extensive study, we studied the endoscopical biopsy specimens and serum hs-CRP levels of 811 patients with chronic gastritis.

We indicated that serum hs-CRP level increases in patients with chronic gastritis and this could be an indicator of severity of acute or chronic mucosal inflammation, and presence of HP infection. Therefore, hs-CRP level may aid the diagnosis of chronic gastritis, but is not usefull for predicting cancer screening or precancerous lesions.

\section{References}

1 Ruggiero P. Helicobacter pylori and inflammation. Curr Pharm Des. 2010;16:4225-36. doi: $10.2174 / 138161210794519075$.

2 Watari J, Chen N, Amenta PS, Fukui $\mathrm{H}$, Oshima T, Tomita T, Miwa H, Lim KJ, Das KM. Helicobacter pylori associated chronic gastritis, clinical syndromes, precancerous lesions, and pathogenesis of gastric cancer development. World J Gastroenterol. 2014;20:5461-73. doi: 10.3748/wjg.v20. i18.5461.

3 Dixon MF, Genta RM, Yardley JH, Correa $P$. Classification and grading of gastritis. The updated Sydney system. International workshop on the histopathology of gastritis, Houston 1994. Am J Surg Pathol. 1996;20:1161-81. doi: 10.1097/00000478199610000-00001.

4 Leung WK, Lin SR, Ching JY, To KF, Ng EK, Chan FK, Lau JY, Sung JJ. Factors predicting progression of gastric intestinal metaplasia: results of a randomised trial on Helicobacter pylori eradication. Gut. 2004;53:1244-9. doi: 10.1136/gut.2003.034629.

5 Saribas S, Kocazeybek B, Aslan M, Altun $S$, Seyhun Y, Oner YA, Memisoglu N. Do procalcitonin and C-reactive protein levels have a place in the diagnosis and followup of Helicobacter pylori infections? J Med
Microbiol. 2004;53:639-44. doi: 10.1099/ jmm.0.05398-0.

6 Raut SC, Patil VW, Dalvi SM, Bakhshi GD. Helicobacter pylori gastritis, a presequeale to coronary plaque. Clin Pract. 2015;5:717. doi: 10.4081/cp.2015.717.

7 Farah R, Hamza H, Khamisy-Farah R. A link between platelet to lymphocyte ratio and Helicobacter pylori infection. J Clin Lab Anal. 2018 Jan;32(1). doi: 10.1002/jcla.22222.

8 Farah R, Khamisy-Farah R. Association of neutrophil to lymphocyte ratio with presence and severity of gastritis due to Helicobacter pylori infection. J Clin Lab Anal. 2014;28:219-23. doi: 10.1002/jcla.21669.

9 Kolb-Bachofen V. A review on the biological properties of C-reactive protein. Immunobiology. 1991;183:133-45. doi: 10.1016/S0171-2985(11)80193-2.

10 Musunuru K, Kral BG, Blumenthal RS, Fuster V, Campbell CY, Gluckman TJ, Lange RA, Topol EJ, Willerson JT, Desai MY, Davidson MH, Mora S. The use of high-sensitivity assays for C-reactive protein in clinical practice. Nat Clin Pract Cardiovasc Med. 2008;5:62135. doi: 10.1038/ncpcardio1322.

11 Chung HW, Kim JW, Lee JH, Song SY, Chung JB, $\mathrm{Kwon} \mathrm{OH}$, Lim JB. Comparison of the validity of three biomarkers for gastric cancer screening: carcinoembryonic antigen, pepsinogens, and high sensitive C-reactive protein. J Clin Gastroenterol. 2009;43:1926. doi: 10.1097/MCG.0b013e318135427c.

12 Rahmani A, Moradkhani A, Hafezi Ahmadi MR, Jafari Heirdarlo A, Abangah G, Asadollahi K, Sayehmiri K. Association between serum levels of high sensitive C-reactive protein and inflammation activity in chronic gastritis patients. Scand J Gastroenterol. 2016;51:531-7. doi: 10.3109/00365521.2015.1102318.

13 Wang P, Ji R, Yu T, Zuo XL, Zhou CJ, Li CQ, Li Z, Li YQ. Classification of histological severity of Helicobacter pylori-associated gastritis by confocal laser endomicroscopy. World J Gastroenterol. 2010;16:5203-10. doi: 10.3748/wjg.v16.i41.5203.

14 Faigel DO, Furth EE, Childs M, Goin J, Metz DC. Histological predictors of active Helicobacter pylori infection. Dig Dis Sci. 1996;41:937-43. doi: 10.1007/BF02091534.

15 Sepulveda AR, Patil M. Practical approach to the pathologic diagnosis of gastritis. Arch 
Pathol Lab Med. 2008;132:1586-93. PMDI: 18834216.

16 Wong BC, Lam SK, Wong WM, Chen JS, Zheng TT, Feng RE, Lai KC, Hu WH, Yuen ST, Leung SY, Fong DY, Ho J, Ching CK, Chen JS; China Gastric Cancer Study Group. Helicobacter pylori eradication to prevent gastric cancer in a high-risk region of China: a randomized controlled trial. JAMA. 2004;291:187-94. doi: 10.1001/jama.291.2.187.

17 Ford AC, Forman D, Hunt R, Yuan Y, Moayyedi P. Helicobacter pylori eradication for the prevention of gastric neoplasia. Cochrane Database Syst Rev. 2015;7:CD005583. PMDI: 26198377.

18 Chen Z, Huang Y, Li S, Lin J, Liu W, Ding Z, Li $X$, Chen $Y$, Pang W, Yang D, Su Z, Li J, Weng $Y$, Zhang $X$. Platelet-to-white blood cell ratio: a prognostic predictor for 90-day outcomes in ischemic stroke patients with intravenous thrombolysis. J Stroke Cerebrovasc Dis. 2016;25:2430-8. doi: 10.1016/j. jstrokecerebrovasdis.2016.06.015.

19 Stolte M, Meining A. The updated Sydney system: classification and grading of gastritis as the basis of diagnosis and treatment. Can J Gastroenterol. 2001;15:591-8. doi: 10.1155/2001/367832.

20 Ernst Peter B, Gold Benjamin D. The disease spectrum of Helicobacter pylori: the immunopathogenesis of gastroduodenal ulcer and gastric cancer. Annu Rev Microbiol. 2000;54:615-40. doi: 10.1146/annurev. micro.54.1.615.

21 Leontiadis, Grigoris I, Sharma Virender K, Howden Colin W. Non-gastrointestinal tract associations of Helicobacter pylori infection: what is the evidence? Arch Intern Med. 1999;159(9):925-40. PMID: 10326935.

\section{Correspondence:}

Dr. Eren Altun

Balikesir Universty, Faculty of Medicine, Department of Pathology

Balikesir Turkey

Phone: +90 5303411651

erenaltun@hotmail.com

Received: Nov 06, 2018

Review: Jan 10, 2019

Accepted: Feb 03, 2019
Conflict of interest: none

Financial source: none
${ }^{1}$ Research performed at Balikesir Universty, Faculty of Medicine, Department of Pathology, Balikesir, Turkey.

